\title{
Yang-Lee Zeros of a Planar Ising Model with a Boundary Magnetic Field
}

\author{
D. B. Abraham^ and J. De Coninck \\ Université de l'Etat à Mons, Faculté des Sciences, B-7000 Mons, Belgium
}

\begin{abstract}
A planar Ising ferromagnet is investigated with a magnetic field acting on one surface. The Yang-Lee zeros associated with this field are located exactly on the imaginary axis and their limiting distribution is given. Above the critical temperature, this distribution has a gap, near which the pair correlation for spins in the surface exhibits critical behaviour. The zeros of certain antiferromagnets are located, in particular those for an antiferromagnetic ring coupled ferromagnetically to a planar Ising ferromagnet.
\end{abstract}

\section{Introduction}

Since the seminal work of Yang and Lee [1,2], the location of zeros of partition functions, in particular its limiting behaviour for systems of infinite volume, has been a cornerstone in the statistical-mechanical treatment of phase transitions. For instance, in the Ising ferromagnet with pair interactions and an applied magnetic field $h$ (in units of $k_{B} T$ ) acting on all spins, no matter what the integer dimension $d$ is, the zeros all lie on the imaginary $h$ axis [2]. The generally accepted picture is:

1. For $T>T_{c}(d), T_{c}(d)$ being the d-dimensional critical temperature, there is a window $\left(-i h_{g}, i h_{g}\right)$ uniformly free of zeros. As $T \rightarrow T_{c}(d)+, h_{g} \sim\left(T-T_{c}(d)\right)^{\Delta}$, where $\Delta$ is the usual gap exponent. For $T<T_{c}(d)$, there is a non-zero density of zeros at $h=0$ in the infinite volume limit.

2. The density $G(i h, T)$ of zeros has a branch point singularity near $h_{g}$ of the type

$$
G(i h, T) \sim\left(h-h_{g}\right)^{\sigma},
$$

so that the magnetisation, which is essentially the Hilbert transform of $G$ as a function of $h$, has the same singularity structure. It is periodic, period $2 \pi i$ and analytic in the strip $-\pi<\operatorname{Im} h<\pi$ provided it is cut on the imaginary $h$ axis, \pm $\left(h_{g}, \pi\right) \bmod 2 \pi$.

\footnotetext{
* On leave from: University of Oxford. Current Address: Department of Mathematics, The University of
} Texas at Austin, Austin, Texas 78712, USA 
3. It is tempting to regard the Yang-Lee edge singularity as a critical point in its own right $[3,4]$, albeit controlled by a single "thermo-dynamic" parameter. In the vicinity of the edge we therefore anticipate scaling behaviour of the correlation functions as well.

4. Kortman and Griffiths [3] estimated $\sigma$ from high-temperature expansions obtaining $\sigma \sim-1 / 8(d=2)$ and $\sigma \sim+1 / 8(d=3)$ for the spin $-1 / 2$ Ising ferromagnet. The result for $d=1$ is known exactly: $\sigma=-1 / 2$ from the transfer matrix for nearestneighbour coupling. Universality for $d=1$ has been investigated in depth by Fisher [5]: the picture described in 1 through 3 above is rigorously confirmed. For the spherical model, Fisher and Kurtze [6] obtained $G(i h, T)$, with $\sigma=1 / 2$, from the analytic continuation of the infinite volume free energy, but did not confirm the existence of a uniformly zero-free window for finite systems.

5. The Yang-Lee edge singularity has been linked to the following problems:

(i) $\phi^{3}$ field theory and an associated renormalization group scheme $[4,7]$.

(ii) Directed lattice animal enumeration [7] via (i).

(iii) The critical behaviour of branched polymers [8], again via (i).

(iv) Recently, exact relationship between certain $d=3$ directed animal enumerations and $d=2$ extended hard core lattice gases have been established by Dhar [9] giving $\sigma=-1 / 6$ for $d=2$ (compare 4 above) from Baxter's solution of the hard-hexagon problem [10].

The only model, however, for which points 1 to 3 have been established is the $d=1$ Ising ferromagnet, which has a somewhat arcane phase transition at $T=0$. In Sect. 2 of this paper we give results for a field applied to one boundary of a rectangular Ising lattice wrapped on a cylinder whose length is then taken to infinity, but whose circumference has any finite value. This extends exact information obtained by McCoy and $\mathrm{Wu}[11]$ on the equation of state of this model in the thermodynamic limit. Only the limiting density of zeros follows from their calculation, a point made quite clear by the work on temperature zeros by Fisher [12] and by Brascamp and Kunz [13]. Section 3 is devoted to the study of the motion of the Lee-Yang zeros for a finite system with respect to the $z=1$ point of the fugacity plane and to the determination of the limiting density as the total number of spin becomes infinite. The corresponding edge singularity is developed in Sect. 4. We finally locate the zeros of this model when it becomes antiferromagnetic (Sect. 5). The case of an antiferromagnetic ring coupled to a ferromagnetic cylinder is also studied.

\section{The Model and the Corresponding Yang-Lee Zeros}

Consider a lattice $\Lambda_{N} \subset \mathbb{Z}^{d}$, where $x \in \Lambda_{N}$ means $1 \leqq x_{i} \leqq N$ for $i=1, \ldots, d, d$ being the spatial dimensionality. At each vertex of such a lattice there is a spin $\sigma(i)= \pm 1$. The collection of spins has an energy

$$
E_{\Lambda}(\{\sigma\})=-\sum_{i, j \in \Lambda} J(i, j) \sigma(i) \sigma(j)-H \sum_{i \in \Lambda} \sigma(i),
$$

and the partition function is

$$
Z_{\Lambda}(h, T)=\sum_{\{\sigma\}} \exp \left(-E_{\Lambda}(\{\sigma\}) / k_{B} T\right),
$$


where $k_{B}$ is the Boltzmann constant and $T$ is the absolute temperature. Clearly $Z_{\Lambda}(h, T)$ is proportional to a polynomial of degree $N(\Lambda)$, the number of sites in $\Lambda_{N}$, in $\exp (2 h)$ with $h=H / k_{B} T$; this polynomial has non-negative coefficients and therefore no real positive zeros.

In this paper we consider a special case of (2) and (3) with $d=2$ and nearestneighbour interactions on a cylinder with a field applied only to spins on one "surface":

$$
\begin{aligned}
E_{\Lambda}(\{\sigma\})= & -\sum_{j=1}^{M}\left[\sum_{i=1}^{N-1} J_{1} \sigma(i, j) \sigma(i+1, j)\right. \\
& \left.+\sum_{i=1}^{N} J_{2} \sigma(i, j) \sigma(i, j+1)+H \sigma(1, j)\right] .
\end{aligned}
$$

We then consider a suitably normalized partition function (3),

$$
f_{M, N}(h, T)=Z_{\Lambda}(h, T) / Z_{\Lambda}(0, T) .
$$

This is up to a factor a polynomial of degree $M$ in $\exp (2 h)$, with non-negative coefficients so that the entire Lee-Yang procedure is again pertinent. Normally, for instance with toroidal boundary conditions, one gets 4 terms, 2 from the "odd" and 2 from the "even" fermion number subspaces denoted $h_{+}$and $h_{-}$. As can be seen, $|+\rangle$ projects onto both $h_{+}$and $h_{-}$. The free boundary condition at the other edge eliminates the projection onto $h_{-}$. The standard techniques of Schultz et al [14] and the ghost spin method [15] reduce (5) to a single product:

$$
\begin{aligned}
f_{M, N}(h, T)=\prod_{\omega>0}^{<\pi}\left\{\cosh ^{2}(h)+\sinh ^{2}(h) \operatorname{cotan}(\omega / 2) \exp \left(2 K_{1}^{*}\right) \times\right. \\
\left.\times \frac{\sinh (N \gamma(\omega)) \sin \left(\delta^{\prime}(\omega)\right)}{\cosh (N \gamma(\omega))+\sinh (N \gamma(\omega)) \cos \left(\delta^{\prime}(\omega)\right)}\right\}
\end{aligned}
$$

with $\exp \left(2 K_{1}^{*}\right)=\operatorname{cotanh}\left(K_{1}\right)$ and

$$
\begin{aligned}
& \tan \left(\delta^{\prime}(\omega) / 2\right)= \sinh \left(2 K_{2}\right) \sin \omega /(\sinh (\gamma(\omega)) \\
&\left.+\sinh \left(2 K_{1}^{*}\right) \cosh \left(2 K_{2}\right)-\sinh \left(2 K_{2}\right) \cosh \left(2 K_{1}^{*}\right) \cos \omega\right), \\
& \cosh (\gamma(\omega))=\cosh \left(2 K_{1}^{*}\right) \cosh \left(2 K_{2}\right)-\sinh \left(2 K_{1}^{*}\right) \sinh \left(2 K_{2}\right) \cos \omega,
\end{aligned}
$$

where $\gamma(\omega) \geqq 0$ for real $\omega$. Finally the product in (6) is over $\omega$ such that exp $(i \omega M)=-1$; i.e. $\omega=(2 j-1) \pi / M$, where $j=1, \ldots, M ; \omega=0$ is never allowed, but $\omega=\pi$ is for $M$ odd.

The zeros of the partition function corresponding to (4) are obviously those of the factors of (6). They are thus given by $h=i \psi / 2$, where

$$
\begin{aligned}
\operatorname{cotan}^{2}(\psi / 2)= & \operatorname{cotan}(\omega / 2) \exp \left(2 K_{1}^{*}\right) \tan \left(\delta^{\prime}(\omega) / 2\right) \\
& \cdot \frac{1-\exp (-2 N \gamma(\omega))}{1+\tan ^{2}\left(\delta^{\prime}(\omega) / 2\right) \exp (-2 N \gamma(\omega))},
\end{aligned}
$$

which has $M$ real solutions for $\psi \in(0,2 \pi)$ with $\exp (i M \omega)=-1$. The fact that the zeros in $h$ are pure imaginary, the Lee-Yang property, is not surprising: if a field $h^{\prime}$ is 
applied to the remaining spins in the system then for $(\operatorname{Re} h)$ and $\left(\operatorname{Re} h^{\prime}\right)>0$, $Z_{\Lambda}\left(h, h^{\prime}, T\right) \neq 0$. An intuitive continuity argument on taking $h^{\prime}$ to zero can be made rigorous by deploying Hurwitz's theorem, as shown by Simon [24].

\section{Behaviour of the Yang-Lee Zeros}

The exact determination of the Yang-Lee zeros allows us to give a complete description of their behaviour. We are mainly interested in the motion of the zeros with respect to the $z=1$ point in the fugacity plane not only for those which admit a density, but for all of them.

For simplicity, let us consider the semi-infinite cylinder case, i.e. when the number of rows becomes infinite. Using (7) and (9), the Yang-Lee zeros are simply given by

$$
\begin{aligned}
\omega & =(2 j-1) \pi / M, \\
\operatorname{cotan}^{2}(\psi / 2) & =F(\omega),
\end{aligned}
$$

where

$$
\begin{aligned}
F(\omega)= & \exp \left(2 K_{1}^{*}\right) \sinh \left(2 K_{2}\right)(1+\cos \omega) /(\sinh (\gamma(\omega)) \\
& \left.+\sinh \left(2 K_{1}^{*}\right) \cosh \left(2 K_{2}\right)-\sinh \left(2 K_{2}\right) \cosh \left(2 K_{1}^{*}\right) \cos \omega\right) .
\end{aligned}
$$

The right-hand side is non-negative, monotone non-increasing in $\omega \in(0, \pi]$, even and with period $2 \pi$ in $\omega$. For all $T, F(\pi)=0$; thus when $M$ is odd, $\psi=\pi(\bmod 2 \pi)$ is a zero. The remaining zeros are symmetrical in the $\exp (\mathrm{i} \psi)$ plane with respect to the real axis.

The value $\omega=0$ is never allowed $\left(\omega_{\min }=\pi / M\right)$ but the behaviour of $F(\omega)$ as $\omega+0$ must be considered carefully. For $T>T_{c}$,

$$
F(0)=\exp \left(2 K_{1}^{*}\right) \sinh \left(2 K_{2}\right) / \sinh (\gamma(0)),
$$

giving a uniformly zero-free window $\left[-\psi_{0},+\psi_{0}\right]$, where

$$
\operatorname{cotan}\left(\psi_{0} / 2\right)=\sqrt{F(0)}
$$

In the case $K_{1}=K_{2}$, it is easy to see that $\psi_{0}$ is monotone decreasing as the temperature decreases. As a function of $M$, the nearest zero is

$$
\psi_{0}(M)=\psi_{0}-\frac{\left(1-\cos \left(\psi_{0}\right)\right)^{2}}{2 \sin \left(\psi_{0}\right)} F^{\prime \prime}(0)\left(\frac{\pi}{M}\right)^{2}+O\left(\frac{1}{M^{4}}\right) .
$$

On the other hand, when $T<T_{c}$ the behaviour is quite different: $F(\omega) \rightarrow+\infty$ as $\omega \rightarrow 0$. This means that the zeros close onto the real axis as $M \rightarrow \infty$ :

$$
\psi_{\min }(M)=(2 / \sqrt{B}) \frac{\pi}{M}\left(1+O\left(\frac{1}{M^{2}}\right)\right),
$$

where

$$
B=\lim _{\omega \rightarrow 0} \omega^{2} F(\omega)
$$


Precisely at $T=T_{c}$, yet another behaviour is given:

$$
\psi_{\min }(M) \sim 2\left\{2 \exp \left(2 K_{2}(c)\right) \sqrt{\sinh \left(2 K_{2}(c)\right)}\right\}^{-1 / 2}\left(\frac{\pi}{M}\right)^{1 / 2}\left(1+O\left(\frac{1}{M^{2}}\right)\right),
$$

where $K_{2}(c)$ simply means that we are at the critical temperature. This agrees with Itzykson et al's scaling hypothesis for the zeros [17].

Let the zero corresponding to $\omega=(2 j-1) \pi / M$ be labelled $\psi_{j}(M)$. Then if $j$ is held fixed and $M$ is increased, $\psi_{j}(M)$ is monotone decreasing. This confirms a conjecture of Griffiths and Nishimori on the "first zero" (nearest to the positive real axis) in this case [18].

We now consider the limiting zeros density.

As $N \rightarrow \infty$, one gets from (6),

$$
f_{M, \infty}(h, T)=\prod_{\omega>0}^{<\pi}\left[\cosh ^{2}(h)+\sinh ^{2}(h) \operatorname{cotan}^{2}(\psi(\omega) / 2)\right]
$$

The mean value of the magnetisation variable can therefore be written as

$$
\partial_{h} \log f_{M, \infty}(h, T)=\sum_{\omega>0}^{<\pi} \frac{\sinh (2 h)\left(1+\operatorname{cotan}^{2}(\psi(\omega) / 2)\right)}{\cosh ^{2}(h)+\sinh ^{2}(h) \operatorname{cotan}^{2}(\psi(\omega) / 2)}
$$

which, in terms of the Yang-Lee variable $z=\exp (-2 h)$, leads to

$$
\partial_{h} \log f_{M, \infty}(h, T)=2\left(1-z^{2}\right) \sum_{\omega>0}^{<\pi} \frac{1}{z^{2}-2 \cos (\psi(\omega)) z+1} .
$$

As the number of rows becomes infinite, the magnetisation per spin $m(h, T)$ can be computed as

$$
m(h, T)=\lim _{M \rightarrow \infty} \frac{1}{M} \partial_{h} \log f_{M, \infty}(h, T)
$$

i.e.

$$
m(h, T)=\frac{1}{\pi} \int_{0}^{\pi} d \omega \frac{\left(1-z^{2}\right)}{z^{2}-2 \cos (\psi(\omega)) z+1},
$$

since this integral obviously exists. It remains to change the variable of integration to let the limiting density of zeros appear:

where, formally,

$$
m(h, T)=\int_{0}^{\pi} d \psi g(\psi) \frac{\left(1-z^{2}\right)}{z^{2}-2 \cos (\psi) z+1},
$$

$$
g(\psi)=\frac{1}{\pi} \frac{d \omega}{d \psi}
$$

on the limiting solution set of (11), zero elsewhere. The existence of this density is guaranteed by the existence and the sign of the derivative

$$
\frac{d \psi}{d \omega}=\frac{-1}{1+F(\omega)} \cdot \frac{F^{\prime}(\omega)}{\sqrt{F(\omega)}},
$$

where the prime denotes derivation with respect to $\omega$. 
For $T>T_{c}$, where $T=T_{c}$ is the unique real solution of $K_{1}^{*}=K_{2}$, define the gap $\theta_{g}$ by

$$
\theta_{g}=\sup _{\theta \in[0, \pi]}\{\theta: g(\theta)=0\}
$$

Then one gets

$$
\tan ^{2}\left(\theta_{g} / 2\right)=\exp \left(-2 K_{1}^{*}\right) \sinh (\gamma(0)) / \sinh \left(2 K_{2}\right)
$$

As $T \rightarrow T_{c}+$, one easily gets from this relation

$$
\theta_{g} \sim\left(T-T_{c}\right)^{1 / 2}
$$

giving a gap exponent $\Delta=1 / 2$.

Whenever $T>T_{c}$, we now turn to the "edge-singularity" of the density $g(\theta)$. Up to the second order in $\omega$, one has

$$
\operatorname{cotan}^{2}(\theta / 2)=F(0)+\frac{1}{2} F^{\prime \prime}(0) \cdot \omega^{2} .
$$

Since for $\theta \gtrsim \theta_{g}$,

$$
\operatorname{cotan}^{2}(\theta / 2)=\operatorname{cotan}^{2}\left(\theta_{g} / 2\right)-\operatorname{cotan}\left(\theta_{g} / 2\right) \sin ^{-2}\left(\theta_{g} / 2\right)\left(\theta-\theta_{g}\right)+O\left(\theta-\theta_{g}\right)^{2},
$$

one obtains

$$
|\omega|=\left(\frac{\operatorname{cotan}\left(\theta_{g} / 2\right)}{\sin ^{2}\left(\theta_{g} / 2\right) F^{\prime \prime}(0)}\right)^{1 / 2}\left(\theta-\theta_{g}\right)^{1 / 2}\left(1+O\left(\theta-\theta_{g}\right)\right)
$$

so that, using (25)

$$
g(\theta) \sim\left(\theta-\theta_{g}\right)^{-1 / 2}
$$

This is like the $d=1$ Ising ferromagnet (with $T_{c}=0$ ) and unlike the spherical model [6].

For $T<T_{c}$, we are particularly interested in the behaviour of the density at the origin. Since one has within this regime of temperature

$$
F(\omega)=\frac{4 \exp \left(2 K_{1}^{*}\right) \sinh \left(2 K_{2}\right)}{\left[\cosh (\gamma(0)) \cdot \gamma^{\prime \prime}(0)+\sinh \left(2 K_{2}\right) \cosh \left(2 K_{1}^{*}\right)\right] \omega^{2}}\left(1+O\left(\omega^{2}\right)\right),
$$

one easily deduces from (25) and (26) that

$$
\lim _{\theta \rightarrow 0} g(\theta)=\frac{1}{\pi}\left\{\frac{\exp \left(2 K_{1}^{*}\right) \sinh \left(2 K_{2}\right)}{\operatorname{cotanh}(\gamma(0)) \cdot \sinh \left(2 K_{1}^{*}\right) \sinh \left(2 K_{2}\right)+\sinh \left(2 K_{2}\right) \cosh \left(2 K_{1}^{*}\right)}\right\}^{1 / 2}
$$

which is as expected different from zero. At $T=T_{c}$, the same kind of calculations leads to

$$
g(\theta) \sim|\theta| .
$$

Remark. We now would like to stress the connection existing between the behaviour of the first Yang-Lee zero and the nature of the scaling limit at the critical point. For the model we consider in this paper, one of us (DBA) has indeed already calculated 
the critical point limit law for the magnetisation variable suitably renormalized. The result is the following [15]:

$$
\lim _{M \rightarrow \infty}\left\langle\exp \left(u \sum_{k=1}^{M} \sigma_{1 k}\right) /(M \log M)^{1 / 2}\right\rangle=\exp \left(C u^{2} / 2\right)
$$

for any real $u$, where $C$ is some positive constant and where the mean value $\langle>$ is to be taken with respect to (4) at the critical point as $N \rightarrow \infty$. One gets using the YangLee theorem combined with the Hadamard's representation of an entire function [19]:

$$
\left\langle\exp \left(u \sum_{k=1}^{M} \sigma_{1 k}\right)\right\rangle=\prod_{1}^{\infty}\left(1+\left(u^{2} / u_{j}^{2}\right)\right)
$$

where $u_{1}^{2} \leqslant u_{2}^{2} \leqq \cdots$ are the Yang-Lee zeros given, modulo $2 \pi$, by (10-12). Therefore

$$
\left\langle\exp \left(u \sum_{k=1}^{M} \sigma_{1 k}\right) /(M \log M)^{1 / 2}\right\rangle=\prod_{1}^{\infty}\left(1+\frac{u^{2}}{u_{j}^{2} \cdot M \log M}\right) .
$$

Let us assume that as $M \rightarrow \infty$, the sequence of reduced variables $\sum_{k=1}^{M} \sigma_{1 k} /(M \log M)^{1 / 2}$ converges in distribution to some variable $X$. One obtains that the moment generating function of $X$ has to be entire of order between 1 and 2, with variance unity. The fact that the order $\rho$ has to be between 1 and 2 follows by use of the Lévy's theorem $[20](\rho \geqq 1)$ and of the usual Gaussian domination $(\rho \leqq 2)$. The other properties can be proved using standard probabilistic techniques. This means that, for any $u \in \mathbb{R}$ :

$$
E\{\exp (u X)\}=\exp \left(C u^{2}\right) \cdot \prod_{1}^{\infty}\left(1+u^{2} / C_{j}^{2}\right)
$$

with $\sum_{1}^{\infty} C_{j}^{-2}<+\infty$. Hurwitz's theorem and Newman's results [23] then guarantees that

$$
u_{j}^{2} M \log M \longrightarrow C_{j}^{2} \quad \text { as } \quad M \rightarrow \infty
$$

Since one has proved in (18) that

$$
u_{1}^{2} \sim \frac{C^{\underline{t e}}}{M}
$$

one easily deduces from (40) and (41) that the scaling limit has to be Gaussian.

Whether or not the first Yang-Lee zero verifies some universal property such as suggested for instance by Aizenman's result [21] remains an open interesting question.

\section{The Yang-Lee Edge Singularity}

Kortman and Griffiths [3] and Fisher [4] have stressed the significance of the edge singularity: for fields near the edge, the system is expected to display critical 
fluctuations. To investigate this, we have calculated the magnetisation and pair correlation function between spins on the edge for any complex value of edge field [11]. Our results are

$$
m(h)=\exp \left(K_{2}\right) \frac{1}{\pi} \int_{0}^{\pi} d \omega A^{-1}(\omega)
$$

and

$$
\langle\sigma(1,1) \sigma(1,1+s)\rangle-m^{2}(h)=I_{+} I_{-}+J_{+} J_{-},
$$

where

$$
\begin{gathered}
A(\omega)=\left\{\cosh \left(2 h^{*}\right)-\cos (\omega) \sinh \left(2 h^{*}\right)\right\} \exp \left(K_{2}\right) \\
+\sin (\omega) \sinh \left(2^{*}\right) \exp \left(-K_{2}\right) \tan (\delta /(\omega) / 2), \\
\tanh (\delta /(\omega) / 2)=\sinh \left(2 K_{1}^{*}\right) \sin (\omega) /\left[\exp (\gamma(\omega))-\exp \left(-2 K_{2}\right)\right. \\
\left.\times\left(\cosh \left(2 K_{1}^{*}\right)+\sinh \left(2 K_{1}^{*}\right) \cos (\omega)\right)\right], \\
I_{ \pm}=\frac{1}{2 \pi} \int_{-\pi}^{\pi} d \omega \exp (i \omega s) A^{-1}(\omega)\left[\operatorname { e x p } \left(-\left(K_{2}\right) \cosh \left(2 h^{*}\right) \sin (\omega) \tan \left(\delta^{*}(\omega) / 2\right)\right.\right. \\
\left.+\exp \left(K_{2}\right) \sinh \left(2 h^{*}\right)-\cosh \left(2 h^{*}\right) \cos (\omega)\right) \\
+i\left\{\exp \left(K_{2}\right) \sin (\omega) \cosh \left(2 h^{*}\right)+\exp \left(-K_{2}\right) \tan \left(\delta^{*}(\omega) / 2\right)\right. \\
\left.\cdot\left(\cosh \left(2 h^{*}\right) \cos \omega \pm \sinh \left(2 h^{*}\right)\right\}\right], \\
J_{ \pm}=\frac{1}{2 \pi} \int_{-\pi}^{\pi} d \omega \exp (\mp i \omega s) A^{-1}(\omega) \\
\cdot\left[\left\{ \pm \exp \left(K_{2}\right) \cos (\omega) \mp \exp \left(-K_{2}\right) \sin (\omega) \tan \left(\delta^{*}(\omega) / 2\right)\right\}\right. \\
\left.+i\left(\exp \left(K_{2}\right) \sin (\omega)+\exp \left(-K_{2}\right) \cos (\omega) \tan \left(\delta^{*}(\omega) / 2\right)\right\}\right] .
\end{gathered}
$$

Therefore

$$
\langle\sigma(1,1) \sigma(1,1+s)\rangle-m^{2}(h) \sim \exp \left(-\omega_{0} \cdot s\right) G\left(\omega_{0}\right) \text { for } s \omega_{0} \gg 1,
$$

where $G$ does not depend on $s$ and is non-zero, and where

$$
\exp \left(\gamma\left(i \omega_{0}\right)\right)=\exp \left(2 K_{2}\right)\left[\cosh \left(2 K_{1}^{*}\right)-\cosh (2 h) \sinh \left(2 K_{1}^{*}\right)\right],
$$

where the gap is given equivalently by

$$
\exp (\gamma(0))=\exp \left(2 K_{2}\right)\left[\cosh \left(2 K_{1}^{*}\right)-\cosh \left(2 h_{q}\right) \sinh \left(2 K_{1}^{*}\right)\right]
$$

Clearly the inverse correlation length has the asymptotic form

$$
\omega_{0}^{2}=-4 \sinh (\gamma(0)) \sinh \left(2 h_{g}\right) \exp \left(-\gamma(0)+2 K_{2}\right)\left|h_{g}-h\right|
$$

so that the exponent $v^{*}=+1 / 2$. Also $m(h) \sim\left|h_{g}-h\right|^{-1 / 2}$, implying $\delta=-2$ for the magnetisation near the "edge."

The analysis of (43) and (44) is done by locating the zeros of $A(\omega)$ nearest the real axis. There is thus an intriguing connection between this problem and the surface binding - unbinding transition [22], but there it is the low temperature behaviour of $A(\omega)$ with real $h$ which is of interest. 


\section{Antiferromagnetic Zeros}

The same methods give the location of surface zeros for the antiferromagnet with coupling $-J_{1}$ and $-J_{2}\left(J_{1}, J_{2}>0\right)$ at

$$
\begin{aligned}
\tanh ^{2} h= & {\left[\sinh (\hat{\gamma}(\omega))+\sinh \left(2 K_{1}^{*}\right) \cosh \left(2 K_{2}\right)+\sinh \left(2 K_{2}\right) \cosh \left(2 K_{1}^{*}\right) \cos \omega\right] / } \\
& {\left[\exp \left(2 K_{1}^{*}\right) \sinh \left(2 K_{2}\right)(1+\cos \omega)\right], }
\end{aligned}
$$

where $\exp (i M \omega)=1$ and

$$
\cosh (\hat{\gamma}(\omega))=\cosh \left(2 K_{1}^{*}\right) \cosh \left(2 K_{2}\right)+\sinh \left(2 K_{1}^{*}\right) \sinh \left(2 K_{2}\right) \cos \omega
$$

with $\hat{\gamma}(\omega) \geqq 0$ for $\omega$ real. In order to verify that the zeros lie on the negative real axis in the $\exp (2 h)$ plane, typical of the $d=1$ antiferromagnet, one only has to prove that

$$
\cosh (2 h) \leqq-1 .
$$

Using hyperbolic identities, one gets

$$
\cosh (2 h)=-1+2 \frac{\sinh \left(2 K_{2}\right) \exp \left(2 K_{1}^{*}\right)(1+\cos \omega)}{\exp (-\hat{\gamma}(\omega))-\exp \left(2 K_{1}^{*}-2 K_{2}\right)} .
$$

In order to prove the validity of (55), it remains to show that

$$
\exp (-\hat{\gamma}(\omega))<\exp \left(2 K_{1}^{*}-2 K_{2}\right)
$$

which is indeed the case for any finite value of $M$ (cf. (54)).

As a final generalization, the problem of the $d=1$ antiferromagnetic ring with coupling constant $-J_{3}\left(J_{3}>0\right)$ coupled ferromagnetically to a ferromagnetic cylinder with couplings $J_{1}, J_{2}\left(J_{1}, J_{2}>0\right)$ can be analysed. The zeros are given for any finite cylinder $N, M$ by:

$$
\left.\operatorname{cotanh}^{2}(h)=-\operatorname{cotan}(\omega / 2) G(\omega)\right) / H(\omega),
$$

where

$$
\begin{aligned}
G(\omega)= & {\left[\cosh \left(2 K_{3}\right)-\sinh \left(2 K_{3}\right) \cos (\omega)\right] \exp \left(-2 K_{1}^{*}\right) \sin \left(\delta^{\prime}(\omega)\right) \sinh (2 N \gamma(\omega)) } \\
& -\sinh \left(2 K_{3}\right) \sin (\omega)\left[\cosh (2 N \gamma(\omega))+\cos \left(\delta^{\prime}(\omega)\right) \cdot \sinh (2 N \gamma(\omega))\right] \\
H(\omega)= & -\sin (\omega) \sinh \left(2 K_{3}\right) \exp \left(-2 K_{1}^{*}\right) \sin \left(\delta^{\prime}(\omega)\right) \sinh (2 N \gamma(\omega)) \\
& +\left[\cosh \left(2 K_{3}\right)+\sinh \left(2 K_{3}\right) \cos \omega\right] \cdot[\cosh (2 N \gamma(\omega)) \\
& \left.+\cos \left(\delta^{\prime}(\omega)\right) \sinh (2 N \gamma(\omega))\right] .
\end{aligned}
$$

As the number of rows $N$ becomes infinite, this leads to

$$
\begin{gathered}
\operatorname{cotanh}^{2}(h)=-\operatorname{cotan}(\omega / 2) \frac{\left[\cosh \left(2 K_{3}\right)-\sinh \left(2 K_{3}\right) \cos (\omega)\right] \exp \left(-2 K_{1}^{*}\right) \sin \left(\delta^{\prime}(\omega)\right)}{-\sinh \left(2 K_{3}\right) \sin (\omega)\left(1+\sinh \left(2 K_{3}\right) \exp \left(-2 K_{1}^{*}\right) \sin \left(\delta^{\prime}(\omega)\right)+\left[\cosh \left(2 K_{3}\right)\right.\right.} . \\
\left.+\sinh \left(2 K_{3}\right) \cos \omega\right]\left(1+\cos \left(\delta^{\prime}(\omega)\right) .\right.
\end{gathered}
$$

Let us first consider the case $K_{1} \rightarrow \infty$, i.e. when the cylinder becomes more 
ferromagnetic. We then have from (61):

$$
\operatorname{cotanh}^{2}(h)=-\operatorname{cotan}^{2}(\omega / 2)
$$

for any coupling $-J_{3}<0$. The zeros are therefore given by

$$
h=i \omega / 2 \text {. }
$$

They are thus located, as it should, on the unit circle in the $\exp (-2 h)$ plane. However, as $K_{1} \rightarrow 0$, all the zeros move into the real axis:

$$
\operatorname{cotanh}^{2} h=\frac{\sinh \left(2 K_{3}\right)(1+\cos \omega)}{\cosh \left(2 K_{3}\right)+\sinh \left(2 K_{3}\right) \cos \omega} .
$$

In terms of the antiferromagnetic coupling $-J_{3}(<0)$, it can also easily be proved that as $K_{3} \rightarrow 0$, the zeros are on the unit circle and that they move through $-\infty$ as $K_{3} \rightarrow+\infty$.

Acknowledgements. The authors thank G. A. Baker, D. Bessis, F. Dunlop, M. E. Fisher, C. Itzykson, E. H. Leib, P. Moussa, B. Souillard and J-B. Zuber very much for interesting and informative discussions. They also thank CEN Saclay, Melbourne University and Université de l'Etat à Mons for support.

\section{References}

1. Yang, C. N., Lee, T. D.: Statistical theory of equations of state and phase transitions. I. Theory of condensation. Phys. Rev. 87, 404 (1952)

2. Lee, T. D., Yang, C. N.: Statistical theory of equations of state and phase transitions. II. Lattice gas and Ising model. Phys. Rev. 87, 410 (1952)

3. Kortman, P. J., Griffiths, R. B.: Density of zeros on the Lee-Yang circle for two Ising ferromagnets. Phys. Rev. Lett. 27, 1439 (1971)

4. Fisher, M. E.: Lee-Yang edge singularity and $\varphi^{3}$ field theory. Phys. Rev. Lett. 40, 1610 (1978)

5. Fisher, M. E.: Yang-Lee edge behavior in one-dimensional systems. Progr. Theor. Phys. Suppl. 69, $14(1980)$

6. Fisher, M.E., Kurtze, D. A.: The Yang-Lee edge singularity in spherical models. J. Stat. Phys. 19, 205 (1978)

7. Cardy, J. L.: Directed lattice animals and the Lee-Yang edge singularity. J. Phys. A15, L593 (1982)

8. Parisi, G., Sourlas, N.: Critical behavior of branched polymers and the Lee-Yang edge singularity. Phys. Rev. Lett. 46, 871 (1981)

9. Dhar, D.: Exact solution of a directed-site animals-enumeration problem in three dimensions. Phys. Rev. Lett. 51, 853 (1983)

10. Baxter, R. J.: Hard hexagons: Exact solution. J. Phys. A13, L61 (1980)

11. McCoy, B. M., Wu, T. T.: Theory of Toeplitz determinants and the spin correlations in the twodimensional Ising model. IV. Phys. Rev. 162, 436 (1967)

12. Fisher, M. E.: Boulder lectures in theoretical physics, 7C (1964): Boulder: University of Colorado Press

13. Brascamp, H. J., Kunz, H.: Zeros of the partition function for the Ising model in the complex temperature plane. J. Math. Phys. 15, 65 (1974)

14. Schultz, T. D., Mattis, D. C., Lieb, E. H.: Two-dimensional Ising model as a soluble problem of many fermions. Rev. Mod. Phys. 36, 856 (1964)

15. Abraham, D. B.: Block spins in the edge of an Ising ferromagnetic half-plane. J. Stat. Phys. 19, 553 (1978)

16. Lieb, E. H., Sokal, A. D.: A general Lee-Yang theorem for one-component and multicomponent ferromagnets. Commun. Math. Phys. 80, 153 (1981) 
17. Itzykson, C., Pearson, R. B., Zuber, J. B.: Distribution of zeros in Ising and gauge models. Nucl. Phys. B220, 415 (1983)

18. Nishimori, H., Griffiths, R. B.: 'Structure and motion of the Lee-Yang zeros.' preprint 83-017, Rutgers University

19. De Coninck, J., de Gottal, Ph.: New inequalities for Ising ferromagnets. J. Stat. Phys. 36, 181 (1984)

20. Lukacs, E.: 'Characteristic functions' London: M. G. Kendall 1960

21. Aizenman, M.: Geometric analysis of $\Phi^{4}$ fields and Ising models. Parts I and II. Commun. Math. Phys. 86, 1 (1982)

22. Abraham, D. B.: Solvable model with a roughening transition for a planar Ising ferromagnet. Phys. Rev. Lett. 44, 1165 (1980)

23. Newman, C. M.: Critical point inequalities and scaling limits. Commun. Math. Phys. 66, 181 (1979)

24. Simon, B.: 'The $P(\phi)_{n}$ Euclidean quantum field theory.' Princeton, NJ: Princeton University Press, 1974. See pp. 336 and 337

Communicated by M. E. Fisher

Received April 3, 1986 
